\title{
ESTRATEGIAS DE ENSEÑANZA UTILIZADAS EN CLASES DE EVOLUCIÓN BIOLÓGICA
}

\author{
Teaching strategies used in biological evolution classes
}

\author{
Eloy León*
}

\section{RESUMEN}

Las estrategias de enseñanza utilizadas por docentes en las clases de biología para que aprendan los estudiantes son diversas, desde las recomendadas para promover las habilidades cognitivas hasta aquellas que ayudan a comprender los fenómenos naturales. Para el desarrollo de este trabajo, se consideraron las estrategias de enseñanza propuestas por Díaz y Hernández (2002). La investigación tiene un alcance descriptivo. Además, el objetivo específico es identificar las estrategias utilizadas en las clases de evolución biológica por los docentes, ubicados en la ciudad de Maracaibo (Venezuela). Se utilizó el muestreo no probabilístico de tipo por conveniencia, constituida por un total de 24 docentes, que laboran en colegio de media general del sector privado ubicados en la parroquia de Juana de Ávila específicamente en la zona norte de Maracaibo, para los puntos: Avenida Fuerzas Armadas, Milagro norte y Urbanización la Trinidad, tomados en cuenta bajo los siguientes criterios: licenciatura de Educación Mención Biología, localizados en la ciudad de Maracaibo (Venezuela), activos en la labor pedagógica y con un mínimo de dos años de experiencia impartiendo clases de evolución biológica en la materia de Biología a nivel de media general. La técnica utilizada para la recolección de los datos fue una encuesta; empleando el instrumento con escala de estimación, con cuatro alternativas de respuestas. Las ilustraciones y las preguntan intercaladas, tuvieron el mayor porcentaje para la opción Siempre y las gráficas presentaron el mayor porcentaje para la opción Nunca y con una media aritmética más baja.

Palabras claves: Evolución biológica, estrategias de enseñanza, docentes.

RECIBIDO: Agosto 2018

ACEPTADO: Noviembre 2018

\footnotetext{
* Profesor de Biología y Ciencias naturales de la U.E. Udón Pérez. Licenciado en Educación Mención Biología. Maestrante en Enseñanza de la Biología, LUZ. Zulia, Venezuela. Correo electrónico: Eloy88leon@gmail.com
} 


\begin{abstract}
The teaching strategies used by teachers in biology classes for students to learn are diverse, from those recommended to promote cognitive skills to those that help to understand natural phenomena. For the development of this work, the teaching strategies proposed by Díaz and Hernández (2002) were considered. The investigation has a descriptive scope. In addition, its specific objective is to identify the strategies used in classes of biological evolution by teachers, located in the city of Maracaibo (Venezuela). Non-probabilistic type sampling was used for convenience, constituted by a total of 24 teachers, who work in the private high school. Located in the parish of Juana de Avila, specifically in the northern area of Maracaibo, for the following points: Avenida Fuerzas Armadas, Milagro Norte and Urbanización la Trinidad. Taken into account under the following criteria: Bachelor of Education Mention Biology, located in the city of Maracaibo (Venezuela), active in pedagogical work and with a minimum of two years of experience teaching biological evolution classes in the field of Biology at the high school level. The technique used to collect the data was a survey; using the instrument with estimation scale, with four alternative answers. The illustrations and questions interspersed, had the highest percentage for the Always option and the graphs presented the highest percentage for the Never option and with a lower arithmetic mean.
\end{abstract}

Keywords: Biological evolution, teaching strategies, teachers.

\title{
Introducción
}

La enseñanza de la evolución biológica es uno de los temas más discutidos desde que el modelo teórico propuesto por Darwin en 1859 apareciera. Cada vez que se enseña tópicos referidos al contenido evolutivo son numerosos las maneras, técnicas, estrategias o estilos de cómo se puede o debe enseñar. Por ello, surgen una cantidad considerable de formas, guías, orientaciones o ayudas para abordar los temas de la evolución.

Bajo este contexto, las estrategias de enseñanza que los docentes utilizan en clases de biología para que los estudiantes aprendan son diversas, desde aquellas referidas a promover las habilidades cognitivas hasta aquellas que ayudan a la comprensión de los fenómenos naturales, entendiéndose la variable de los grupos de estudiantes y el objeto de estudio. Es decir, que las estrategias son aplicadas a determinados grupos considerando el tipo de contenido que docente va a compartir. 


\section{Eloy León}

Telos Vol. 21, No. 1 (2019). 144-165

En este sentido, los profesores implementan determinadas estrategias de enseñanza conociendo al grupo de estudiantes y al objeto de estudio que será compartido en las clases, partiendo de ello unas estrategias probablemente pueden ser escogidas por unos docentes y otras serán seleccionadas por otros docentes.

Por lo antes mencionado, se consideraron a profesores ubicados en la ciudad de Maracaibo (Venezuela), para abordar la siguiente interrogante: ¿Cuáles estrategias de enseñanza son más utilizadas por los docentes de media general en clases de evolución biológica?

\section{Fundamentación teórica}

\section{Antecedentes de la investigación}

Tapia y Arteaga (2009), realizaron un trabajo de investigación, cuyo objetivo fue; identificar las estrategias utilizadas por docentes de la educación básica para abordar la enseñanza de la célula, contando con un enfoque cualitativo, empleando un tipo de investigación etnográfica educativa, haciendo énfasis en aspectos descriptivos. Con una modalidad de estudio de casos, donde contaron con dos Profesores para las entrevistas y la observación de las clases, las cuales tuvieron un abarque del 9 grado de media general.

La investigación se llevó a cabo en una institución pública en el municipio de Miranda del Estado Zulia. Aplicaron un tipo de observación directa no participante y una entrevista semiestructurada. Para el análisis y la recolección de datos utilizaron una triangulación metodológica; observación de clases, entrevistas y revisión de documentos. En cuanto a la selección de las categorías de análisis, se basaron en las estrategias de enseñanza propuestas por Díaz y Hernández (2002). Por su parte, los investigadores encontraron que la estrategia que prevaleció más en los docentes fueron, preguntas intercaladas, señalizaciones y el discurso oral.

Cabe destacar que, dentro de sus resultados sobresalen dos cuestiones, la primera; las señalizaciones y las preguntas intercaladas, son las estrategias de 
enseñanza más utilizadas en clases de célula, y la segunda; que los profesores no compartieron una variedad de estrategias.

Por otra parte, Vera y Vera (2011), desarrollaron una investigación titulada; Estrategias utilizadas por los docentes para promover el aprendizaje de la biología a nivel universitario, teniendo como objetivo; identificar los tipos de estrategias utilizadas por los docentes para promover el aprendizaje en biología. Llevándose a cabo en la Escuela de Educación, Mención Biología de Universidad del Zulia. Abarcó un alcance de investigación descriptivo, diseño de campo no experimental y transversal, con un método empírico-inductivo.

La población estuvo conformada por 1050 estudiantes, trabajando con una muestra de 91 estudiantes estratificados por semestres, por otra parte, los docentes fueron una población censal de 16 docentes. Implementaron para la técnica y recolección de datos, la encuesta y el instrumento, un cuestionario de 12 ítems. Como referente teórico, utilizaron las estrategias de enseñanza de Díaz y Hernández (2002) y Ausubel et al. (1991). Emplearon la estadística descriptiva mediante la media aritmética.

Dentro de sus resultados, obtuvieron una baja presencia de estrategias para promover el aprendizaje significativo por parte de docentes. Además, las estrategias para generar conocimiento previo y para orientar la atención en el alumnado, tuvo un nivel bajo de aplicación por parte de los docentes.

Bajo este orden de ideas, Acosta y Boscán (2012), realizaron un trabajo de investigación titulado; Estrategias cognoscitivas para la promoción del aprendizaje significativo de la Biología, en la Escuela de Educación, presentando como objetivo, describir las estrategias cognoscitivas que utilizan los docentes para la promoción del aprendizaje significativo de la Biología en la Escuela de Educación. La investigación se desarrolló en la Universidad del Zulia. Siendo su alcance de investigación descriptivo, de campo no experimental y transversal.

La técnica que utilizaron fue la encuesta y el instrumento un cuestionario, aplicado a 6 profesores que socializan sus contenidos en las sub-área diversidad 


\section{Eloy León}

Telos Vol. 21, No. 1 (2019). 144-165

biológica y 357 estudiantes que cursan la carrera Educación Mención Biología, que a su vez están suscritos a las unidades curriculares de la sub-área antes mencionada.

Sus hallazgos, fueron que los docentes de Biología utilizan Casi siempre estrategias cognoscitivas en los contenidos biológicos, lo cual tiene un impacto para promover el aprendizaje significativo. A la par, dichas estrategias, favorecen un pensamiento crítico, complejo y reflexivo. A su vez, los autores destacan en los resultados, que se deben considerar estrategias como la elaboración de resúmenes, y otras, para contribuir en la enseñanza y en el aprendizaje de las ciencias biológicas.

\section{Estrategias de enseñanza}

Para Pimienta (2012), las estrategias son instrumentos de los que el docente se fortalece para favorecer el desarrollo de las competencias académicas en los estudiantes; asimismo y con base a una secuencia didáctica (inicio, desarrollo y cierre), menciona el autor, que la relevancia recae sobre la forma de utilizar e implementar la estrategia en el momento preciso. De este modo, como lo expresa Vera y Vera (2011), también son denominadas estrategias instruccionales, las cuales son utilizadas en varios momentos de una secuencia de enseñanza con la finalidad de lograr una efectividad en el proceso de enseñanza-aprendizaje. Eso quiere decir, que los contenidos aprendidos puedan ser socializados en cualquier contexto de su vida cotidiana. Además, señalan las autoras, que debe evidenciarse una participación activa de los estudiantes para dejar atrás la concepción tradicional del estudiante pasivo.

Por otro lado, Campos (2000), enfatiza diciendo que las estrategias son el arte de proyectar y dirigir; con ella se proyecta, ordena y dirige el mecanismo desarrollar los objetivos establecidos, de modo que, el docente las maneja para medir, promover, facilitar, organizar el proceso del aprendizaje, en pro del proceso de enseñanza. Por su parte, García y Cañal de León (1995), esgrimen las estrategias de enseñanza como un sistema particular conformado por unos determinados tipos de actividades de 
enseñanza los cuales se relacionan a través de unos esquemas organizativos característicos.

En tanto Anijovich y Mora (2009), destaca que las estrategias de enseñanza son un conglomerado de toma de decisiones por parte de profesor para orientar la enseñanza con la finalidad de promover un logro, un saber de aprendizaje en los estudiantes. Es decir, se trata de un lineamiento de orientación general sobre cómo socializar un tópico a estudiar, entendiendo tres cuestiones; qué queremos y por qué y para qué, queremos que comprendan el contenido académico.

A la par, en las estrategias de enseñanza subyacen dos dimensiones, y dentro de estos tres momentos (Ver figura 1) de suma importancia puesto que son episodios relacionados con el pensamiento acerca de qué tipo de estrategia el docente va a socializar y la interacción de la clase. Los autores Anijovich y Mora (2009), denominan a este par de dimensiones, reflexiva; el docente diseña la planificación de la clase, es una dimensión donde incluye el desarrollo cognitivo del docente, desde el análisis del contenido curricular hasta las tomas de decisiones para abordar el tema. Y, acción, acá ocurre la interacción de las decisiones tomadas.

Los momentos, que son elementos presentes en las dimensiones los autores lo describen como: (1) planificación en el que se anticipa la acción; (2) acción propiamente dicha o momento interactivo; (3) evaluar la implementación del curso de acción elegido, es considerado el episodio para meditar acerca de los efectos y resultados durante la labor del docente. 
Figura 1. Dimensiones y momentos en las estrategias de enseñanza.

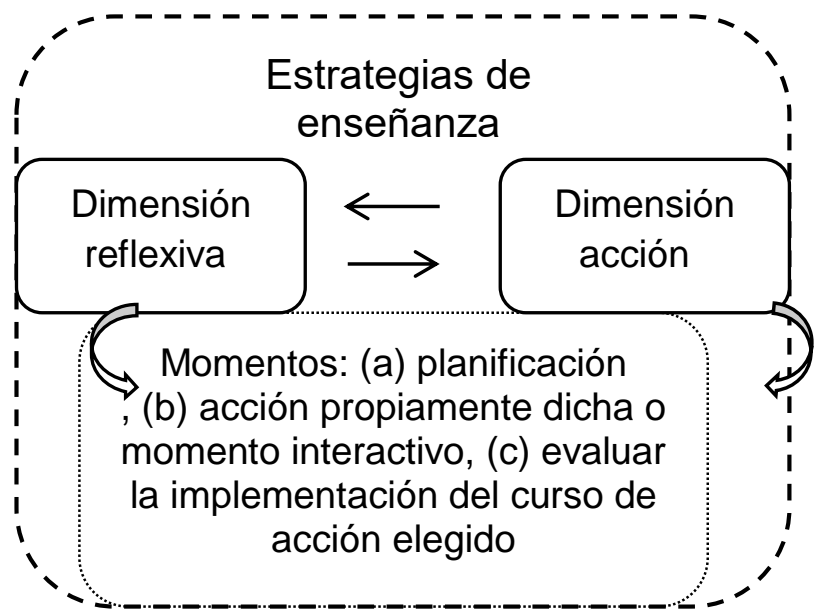

En relación a lo anterior expuesto, cabe considerar lo expresado por Martínez y Zea (2004) y Díaz y Hernández (2002), los cuales formulan unos aspectos a tener en cuenta para determinar qué tipo de estrategia es el indicado para ser socializado en cierto momento de la enseña, a saber:

A.- Características generales de los estudiantes; desarrollo cognitivo, preconcepciones, conocimientos previos, motivación, entre otros.

B.- Tipo de conocimiento en general y del contenido particular, que se desea abordar.

C.- Meta que se desea alcanzar y las actividades cognitivas pedagógicas y afectivas que debe realizar el estudiante para lograrlo.

D.- Retroalimentación constante del proceso enseñanza (de las estrategias de enseñanza utilizadas previamente, si es el caso), asimismo del progreso y aprendizaje de los estudiantes.

E.- Determinación del contexto intersubjetivo (por ejemplo, el conocimiento socializado), generado con los estudiantes hasta ese momento, si es el caso.

F.- Tiempo adecuado para la enseñanza y aprendizaje.

G.- Contar con un diseño de cómo utilizar la estrategia de enseñanza. 
H.- Tener una amplia variedad de estrategias, comprendiendo qué funciones tienen y cómo puede desarrollarse adecuadamente.

Por lo anterior expuesto, cada una de las estrategias de enseñanza que el docente puede utilizar para socializar un tipo de conocimiento científico, debe estar en concordancia con el grupo de estudiantes, es decir con su edad, estatus social, el contexto en el cual cada uno de ellos se desarrolla; debido que es imprescindible, tener en cuenta la realidad del grupo estudiantil y que el contenido que a menudo comparten, es un contenido con un discurso del tipo de conocimiento cotidiano. De allí, toma importancia la creatividad, la manera de abordar un tema y la pertinencia de la estrategia que el docente utiliza.

\section{Tipos de estrategias de enseñanza}

Son numerosas las estrategias de enseñanza con las que el docente puede apoyar sus clases, Díaz y Hernández (2002) sintetizan y clasifica un grupo de estas como las principales compartidas en la dinámica de la enseñanza (Figura 2). A su vez, esgrime que las estrategias de enseñanza se asocian a la programación, elaboración, diseño y realización del contenido temático a aprender por el estudiante, los cuales deberán ser empleados con flexibilidad y adaptados a diversas circunstancias de la enseñanza, por tanto, no deberán de ser rígidos.

Para aplicar las actividades instruccionales, es necesario consideran cuál es el momento oportuno de una clase para utilizar la estrategia. Por ello, vale decir, que existen estrategias de enseñanza que según el momento que sean aplicadas llevan un orden o secuencia didáctica, las del inicio son conocidas como preinstruccionales, las de desarrollo son coinstruccionales y las del cierre son posinstruccionales. 
Figura 2. Estrategias de enseñanza

\begin{tabular}{|c|c|c|}
\hline $\begin{array}{l}\text { Estrategias } \\
\text { enseñanza }\end{array}$ & Descripción & $\begin{array}{l}\text { Efectos y beneficios esperados } \\
\text { en el estudiante }\end{array}$ \\
\hline Objetivos & $\begin{array}{l}\text { Enunciados que establecen } \\
\text { condiciones, tipo de actividad y } \\
\text { forma de evaluación del aprendizaje } \\
\text { del alumno. Como estrategias de } \\
\text { enseñanza compartidas con los } \\
\text { alumnos, generan expectativas } \\
\text { apropiadas. }\end{array}$ & $\begin{array}{l}\text { Dan a conocer la finalidad y } \\
\text { alcance del material y cómo } \\
\text { manejarlo. El alumno sabe qué } \\
\text { se espera de él al terminar de } \\
\text { revisar el material. Ayudan a } \\
\text { contextualizar sus aprendizajes } \\
\text { y a darles sentido. }\end{array}$ \\
\hline Resúmenes & $\begin{array}{l}\text { Síntesis y abstracción de la } \\
\text { información relevante de un discurso } \\
\text { oral o escrito. Enfatizan conceptos } \\
\text { clave, principios y argumento } \\
\text { central. }\end{array}$ & $\begin{array}{l}\text { Facilitan que recuerde y } \\
\text { comprenda la información } \\
\text { relevante del contenido por } \\
\text { aprender. }\end{array}$ \\
\hline $\begin{array}{l}\text { Organizadores } \\
\text { previos }\end{array}$ & $\begin{array}{l}\text { Información de tipo introductorio y } \\
\text { contextual. } \\
\text { Tienden un puente cognitivo entre la } \\
\text { información nueva y la previa. }\end{array}$ & $\begin{array}{l}\text { Hacen más accesible y familiar } \\
\text { el contenido. Con ellos, se } \\
\text { elabora una visión global y } \\
\text { contextual. }\end{array}$ \\
\hline Ilustraciones & $\begin{array}{l}\text { Representaciones visuales de objetos } \\
\text { o situaciones sobre una teoría o tema } \\
\text { específico (fotografías, dibujos, } \\
\text { dramatizaciones, etcétera). }\end{array}$ & $\begin{array}{l}\text { Facilitan la codificación visual } \\
\text { de la información. }\end{array}$ \\
\hline $\begin{array}{l}\text { Organizadores } \\
\text { gráficos }\end{array}$ & $\begin{array}{l}\text { Representaciones visuales de } \\
\text { conceptos, explicaciones o patrones } \\
\text { de información (cuadros sinópticos, } \\
\text { cuadros C-Q-A). }\end{array}$ & $\begin{array}{l}\text { Ayuda a la mejora de los } \\
\text { procesos de recuerdo, } \\
\text { comprensión y aprendizaje sea } \\
\text { por vía textual o escolar. }\end{array}$ \\
\hline Gráficas & $\begin{array}{l}\text { Se trata de recursos que expresan } \\
\text { relaciones de tipo numérico o } \\
\text { cuantitativo entre dos o más factores } \\
\text { o variantes por medio de líneas, } \\
\text { sectores, barras, etcétera. }\end{array}$ & $\begin{array}{l}\text { Ayudan a comprender mejor } \\
\text { las relaciones cuantitativas que } \\
\text { si éstas se expresaran en forma } \\
\text { puramente verbal. Es más, } \\
\text { muchas relaciones cuantitativas } \\
\text { son difíciles de comprender sin } \\
\text { no se utilizan las gráficas }\end{array}$ \\
\hline Analogías & $\begin{array}{l}\text { Proposiciones que indican que una } \\
\text { cosa o evento (concreto y familiar) } \\
\text { es semejante a otro (desconocido y } \\
\text { abstracto o complejo). }\end{array}$ & $\begin{array}{l}\text { Sirven para comprender } \\
\text { información abstractar Se } \\
\text { traslada lo aprendido a otros } \\
\text { ámbitos. }\end{array}$ \\
\hline $\begin{array}{l}\text { Preguntas } \\
\text { intercaladas }\end{array}$ & $\begin{array}{l}\text { Preguntas insertadas en alguna } \\
\text { situación de enseñanza o en un texto. } \\
\text { Mantienen la atención y favorecen la } \\
\text { práctica, la retención y la obtención } \\
\text { de información relevante. }\end{array}$ & $\begin{array}{l}\text { Permite a mantener la atención } \\
\text { y favorece la práctica, la } \\
\text { retención y la obtención de } \\
\text { información importante. }\end{array}$ \\
\hline Señalizaciones & Señalamientos que se hacen en un & practiquen \\
\hline
\end{tabular}




\begin{tabular}{|l|l|l|l|}
\hline & $\begin{array}{l}\text { texto o en la situación de enseñanza } \\
\text { para enfatizar u organizar elementos } \\
\text { relevantes del contenido por } \\
\text { aprender. }\end{array}$ & $\begin{array}{l}\text { consolide lo que ha aprendido. } \\
\text { Mejora la codificación de la } \\
\text { información relevante. El } \\
\text { alumno se autoevalúa } \\
\text { gradualmente. }\end{array}$ \\
\hline $\begin{array}{l}\text { Mapas y redes } \\
\text { conceptuales }\end{array}$ & $\begin{array}{l}\text { Conceptuales } \\
\text { gráficas de esquemas de } \\
\text { conocimiento (indican conceptos, } \\
\text { proposiciones y explicaciones). }\end{array}$ & $\begin{array}{l}\text { Son útiles para realizar una } \\
\text { codificación visual y semántica } \\
\text { de conceptos proposiciones y } \\
\text { explicaciones. } \\
\text { Contextualizar las relaciones } \\
\text { entre conceptos y } \\
\text { proposiciones. }\end{array}$ \\
\hline $\begin{array}{l}\text { Organizadores } \\
\text { textuales }\end{array}$ & $\begin{array}{l}\text { Organizaciones retóricas de un } \\
\text { discurso que influyen en la la } \\
\text { comprensión y el recuerdo. }\end{array}$ & $\begin{array}{l}\text { Facilitan el recuerdo y la } \\
\text { comprensión de las partes más } \\
\text { importantes del discurso }\end{array}$ \\
\hline
\end{tabular}

Fuente: Tomada y adaptado de Díaz y Hernández (2002).

Ahora bien, cada estrategia puede incidir de una manera particular el proceso de enseñanza, de tal modo que Díaz y Hernández (2002), emplean una clasificación teniendo en cuenta los procesos cognitivos activados en los estudiantes durante la estrategia de enseñanza. A saber:

1.- Estrategias para explorar o activar conocimientos previos y estrategias para generar expectativas apropiadas.

Son estrategias que inician las actividades en la secuencia didáctica, motivan al estudiante. Van encaminadas a socializar con la activación de los conocimientos previos, inclusive con generar cuando estos no estén presentes. Asimismo, Indagan acerca de que conocen los estudiantes, de tal modo promover un aprendizaje eficaz. Es decir, buscar los enlaces para relacionar el conocimiento cotidiano con el conocimiento científico o científico escolar.

Según la clasificación de las estrategias de enseñanza de acuerdo al momento de la enseñanza o la secuencia didáctica, la mayoría son estrategias de tipo preinstruccional, tales como: preinterrogatorios, discusión dirigida, enunciación de objetivos, entre otras.

2.- Estrategias para guiar la atención y el aprendizaje.

Son estrategias que se refieren a los recursos que el docente emplea para mantener la atención del estudiante durante el desarrollo de la clase en su entorno 


\section{Eloy León}

Telos Vol. 21, No. 1 (2019). 144-165

académico o escolar. Según la secuencia didáctica, se ubican principalmente en el momento coinstruccional. Algunas de las estrategias que se sugieren son: las señalizaciones, estrategias discursivas orales, entre otras.

3.- Estrategias para mejorar la codificación de la información a aprender.

Son estrategias que se pueden aplicar en cualquier momento de la secuencia didáctica, favoreciendo el desarrollo del aprendizaje. Sin embargo, se utilizan principalmente en el episodio coinstruccional. Se trata de estrategias que van encaminadas a brindar al estudiante la posibilidad de realizar la codificación ulterior, a la expuesta por el docente o el texto escolar. La finalidad es lograr que, la información nueva por aprender presente un mayor nivel de socialización y comprensión. Los autores recomiendas, estrategias como; ilustraciones, organizadores gráficos, señalizaciones, entre otros.

4.- Estrategias para organizar la información nueva por aprender.

Se trata de estrategias para organizar de manera global las ideas contenidas en la información nueva por aprender, posibilitando una correcta coherencia lógica

y mejor significatividad para el aprendizaje de los estudiantes. Pueden emplearse en los tres momentos de la secuencia de enseñanza. Se sugieren estrategias como; mapas conceptuales, resúmenes, organizadores gráficos, cuadro sinóptico, cuadros C-Q-A, entre otras.

5.- Estrategias para promover el enlace entre los conocimientos previos y la nueva información que ha de aprender

Son estrategias destinadas a establecer un enlace entre los conocimientos previos con la información nueva o el contenido científico, de tal manera que ayuda de manera adecuada asegurando una significatividad de los aprendizajes en los estudiantes. A la par, pueden utilizarse en distintos momentos de la secuencia didáctica. Sugieren estrategias tales como; organizadores previos, analogías, organizadores textuales, entre otros. 
Es relevante acotar, que las estrategias de acuerdo al momento en la secuencia didáctica en una clase están orientadas para obtener con el grupo estudiantes un aprendizaje, seguramente a partir de las ideas concebidas, ideas previas o conocimiento previo que poseen estos actores-activos en la dinámica escolar. Así pues, la intención del docente debe ser generar conocimientos en la medida que comparte un saber académico o un contenido de tipo escolar, como el de Biología, siendo del mundo de la ciencia, es utilizado un tipo de conocimiento científico.

En el caso de la Evolución biológica, tópico de conocimiento científico, se pueden emplear una cantidad de estrategias fascinantes; aplicar estudio de casos, museo escolar, debates de modelos teóricos, entre otros (León y Morales, 2017).

\section{Relevancia de la Enseñanza de la Evolución Biológica}

A partir del desarrollo del Modelo Teórico Selección Natural (MTSN), que explica la biodiversidad partiendo de un proceso evolutivo, cuenta Ruiz Gutiérrez et al., (2012), que, desde su difusión a nivel internacional, ha sido indiscutible la necesidad de su enseñanza como una disciplina fundamental, no únicamente para una formación de científicos sino también como parte de la cultura general de la población humana.

Bajo este enfoque y enlazándolo al contexto educativo, Mclnerney (2009) destaca los vínculos con otros temas de los programas académicos, que tiene la evolución biológica, como la Genética: durante las clases de biología cada estudiante constituye una viva demostración de individualidad, que el docente puede aprovechar para hacer la conexión esencial entre selección natural y variación. La genómica comparativa, es posible gracias a las abundantes bases de datos genómicos, ello es una herramienta incuestionable para indagar la relación entre las diferentes formas de vida en la tierra y la secuencia de la prole con modificación; la Salud humana y enfermedad; la Resistencia a antibióticos y el asunto de las Razas.

Bajo el mismo orden de ideas, Futuyma (2005) y León (2017) expresan que debidamente la relevancia que presenta la evolución biológica en distintas áreas de 


\section{Eloy León}

Telos Vol. 21, No. 1 (2019). 144-165

conocimiento o campos de estudio, presentando muchas aplicaciones; contribuye a la medicina y salud pública, la agricultura, la gestión de los recursos naturales, el control de plagas y la conservación de la biodiversidad.

Finalmente, la biología evolutiva conlleva en sí misma una serie de cuestiones que ha ayudado al hombre responder preguntas de gran significado, como lo reseño González Galli (2012), ¿De dónde provenimos? ¿Por qué somos como somos? ¿Qué relación nos une con el resto de las criaturas con las que compartimos este planeta? Y las formulas por Ruiz Gutiérrez et al., (2012), que cuando se enseña y aprende la evolución biológica surgen tales preguntas; cómo se originó los organismos, cómo se diversificaron, a qué se debe que puedan agruparse en dominios, reinos, y el resto de los grupos taxonómicos, cuáles características fenotípicas y genotípicas comparten y cuáles las diferencian, cómo se extinguen las especies, y muchas otras más. Por todo ello, es vasta la cantidad de estrategias que el profesor puede compartir con los estudiantes.

\section{Esquema metodológico}

La investigación contó con un alcance de investigación descriptivo, para identificar las estrategias utilizadas en clases de evolución biológica por parte de los docentes de media general o secundaria. Según señala Hurtado de Barrera (2012), la finalidad de una investigación descriptiva es mostrar el objeto de estudio, a través de una relación, enumeración o categorización minuciosa de sus características, de esta forma, en los resultados podamos alcanzar dos niveles. El primero un nivel elemental, donde se obtenga una sistematización de la información de las características comunes y un segundo nivel más sofisticado, para establecer relación con los elementos observados a fin de obtener un contenido detallado del fenómeno estudiado.

El diseño de la investigación, que atendió el presente trabajo es no experimental, puesto que, las variables de estudio no fueron manipuladas deliberadamente, es decir el objeto de estudio fue estudiado sin variar de manera intencional las variables (Hernández, Fernández y Baptista, 2014). 
La muestra fue de 24 docentes graduados. A la par, fueron consideraron bajo los siguientes criterios: licenciatura de Educación Mención Biología, localizados en la ciudad de Maracaibo (Venezuela), activos en la labor pedagógica, con un mínimo de dos años de experiencia impartiendo clases de evolución biológica en la materia de Biología a nivel de media general.

Para la obtención de dicha muestra, se utilizó el muestreo no probabilístico de tipo por conveniencia, de tal manera, fue posible tener la accesibilidad de los profesores quienes imparten en cursos de secundaria. La distribución de los docentes seleccionados, abarca un total de 7 colegios. Los cuales son de instituciones educativas de media general del sector privado, ubicados en la parroquia de Juana de Ávila específicamente en la zona norte de Maracaibo, para los puntos: Avenida Fuerzas Armadas, Milagro norte y Urbanización la Trinidad.

A continuación, es mostrada una tabla donde aparecen los datos de los docentes.

Tabla 1. Datos de los profesores encuestados referidos a nivel académico y años de experiencia en la docencia.

\begin{tabular}{|c|c|c|c|}
\hline $\begin{array}{c}\text { Cantidad de } \\
\text { docentes }\end{array}$ & Nivel académico & $\begin{array}{l}\text { Cantidad } \\
\text { docentes }\end{array}$ & Años de experiencia \\
\hline \multirow{6}{*}{18} & \multirow{6}{*}{ Licenciatura } & 1 & 3 años \\
\hline & & 1 & 4 años \\
\hline & & 8 & 5 años \\
\hline & & 4 & 6 años \\
\hline & & 2 & 7 años \\
\hline & & 1 & 7 años \\
\hline \multirow{4}{*}{6} & \multirow{4}{*}{ Magister } & 1 & 4 años \\
\hline & & 3 & 5 años \\
\hline & & 2 & 6 años \\
\hline & & 1 & 7 años \\
\hline
\end{tabular}

Fuente: Elaboración propia. 
Eloy León

Telos Vol. 21, No. 1 (2019). 144-165

La técnica utilizada para la recolección de los datos fue una encuesta; empleando el instrumento con escala de estimación, con cuatro alternativas de respuestas: Siempre, Algunas veces, Pocas veces y Nunca.

A la par, fueron estructurados por ítems referidos a: Objetivos, Resúmenes, Organizadores previos, Ilustraciones, Organizadores gráficos, Gráficas, Analogías, Organizadores textuales., Señalizaciones, Mapas y redes conceptuales y Preguntas intercaladas. Además, se elaboró un baremo para la contrastación de los datos obtenidos (Ver tabla 2).

Tabla 2. Baremo

\begin{tabular}{cccc}
\hline $\mathbf{N}$ & Categoría & Rango & Respuesta \\
\hline $\mathbf{1}$ & Muy baja & $1-1,60$ & Nunca \\
$\mathbf{2}$ & Baja & $1,61-2,40$ & Pocas veces \\
$\mathbf{3}$ & Alta & $2,41-3,20$ & Alguna veces \\
$\mathbf{4}$ & Muy alta & $3,21-4$ & Siempre \\
\hline
\end{tabular}

Fuente: Elaboración propia.

\section{Resultados de la investigación}

Luego de recoger los datos, los cuales están vinculados hacia el objetivo de la presente investigación, que es identificar las estrategias más empleadas por docentes de media general en las clases de evolución biológica, se obtuvieron resultados distribuidos en tablas de frecuencias, porcentajes y la media aritmética.

En la siguiente tabla número 3 podemos denotar que los resultados para las estrategias de enseñanza basadas en objetivos presentan porcentaje de un $46 \%$ para la opción de siempre en su utilización en clases de evolución biológica, con una media aritmética de 2,81 que cuando comparamos con la tabla del baremo se puede determinar que tiene una tendencia hacia la categoría Alta. Mientras que, las preguntas intercaladas obtuvieron un alto porcentaje para la opción de Siempre con $71 \%$ y con una media de 3,36 ubicando su aplicación en clases en la categoría de Muy alta.

Entretanto, las Ilustraciones, tienen un porcentaje para la opción siempre con un $71 \%$ y con una media 3,36 con lo cual también están en la categoría de muy alta. 
Por su parte, la utilización de gráficas tiene su mayor valor en la opción Nunca con un $33 \%$, no obstante, al comparar su media de 2,20 están en la categoría de Pocas veces.

Tabla 3. Parte I: Tipos de estrategias de enseñanza utilizadas por docentes de media general.

\begin{tabular}{|c|c|c|c|c|c|}
\hline \multirow[b]{2}{*}{ Estrategias } & \multicolumn{4}{|c|}{ Escala de valor } & \multirow{2}{*}{ Media } \\
\hline & Siempre & $\begin{array}{c}\text { Algunas } \\
\text { veces }\end{array}$ & $\begin{array}{c}\text { Pocas } \\
\text { veces }\end{array}$ & Nunca & \\
\hline \multirow{3}{*}{ Objetivos } & Fi:11 & Fi:5 & Fi: 5 & $\mathrm{Fi}: 3$ & \multirow{3}{*}{2,81} \\
\hline & $\%: 46$ & $\%: 21$ & $\%: 21$ & $\%: 12$ & \\
\hline & Fr: 0,46 & Fr: 0,21 & Fr: 0,21 & Fr:0,12 & \\
\hline \multirow{3}{*}{$\begin{array}{l}\text { Preguntas } \\
\text { intercaladas }\end{array}$} & Fi: 17 & Fi: 5 & $\mathrm{Fi}: 2$ & Fi: 0 & \multirow{3}{*}{3,30} \\
\hline & $\%: 71$ & $\%: 21$ & $\%: 8$ & $\%: 0$ & \\
\hline & Fr: 0,71 & Fr: 0,21 & Fr: 0,08 & Fr: 0 & \\
\hline \multirow{3}{*}{ Ilustraciones } & Fi: 17 & Fi: 7 & Fi: 0 & Fi: 0 & \multirow{3}{*}{3,36} \\
\hline & $\%: 71$ & $\%: 29$ & $\%: 0$ & $\%: 0$ & \\
\hline & Fr: 0,71 & Fr: 0,29 & Fr: 0 & Fr: 0 & \\
\hline \multirow{3}{*}{ Graficas } & Fi: 4 & Fi: 5 & Fi: 7 & Fi: 8 & \multirow{3}{*}{2,20} \\
\hline & $\%: 17$ & $\%: 21$ & $\%: 29$ & $\%: 33$ & \\
\hline & Fr: 0,17 & Fr: 0,21 & Fr: 0,29 & Fr: 0,33 & \\
\hline
\end{tabular}

Fuente: Elaboración propia

Ahora bien, los objetivos están asociados con los tipos de estrategias para activar los conocimientos previos. Siendo influyente en los procesos cognitivos, debido que generan una expectativa en el estudiante, y como lo reseña Díaz y Hernández (2002), deben ser aplicadas con el fin de orientar en los procesos instruccionales. Además, como lo señala Vera y Vera (2011), es fundamental que los docentes puedan esclarecer los objetivos y las actividades acerca del contenido que se desea estudiar. De tal manera, se pueda realizar un primer dialogo y examinar el conocimiento que presentan los estudiantes.

En este orden de ideas, las otras tres estrategias instruccionales están relacionadas con las estrategias para mejorar la codificación de la información a aprender, siendo aplicadas como señala Díaz y Hernández (2002) para consolidar la información o el contenido escolar y que comúnmente son aplicadas durante el momento de desarrollo de la clase, además estas son de gran importancia para enfatizar los conocimientos científicos escolares. En el caso de las Ilustraciones y las 


\section{Eloy León}

Telos Vol. 21, No. 1 (2019). 144-165

gráficas permiten de manera visual apreciar determinados procesos en la naturaleza, comportamiento de fenómenos naturales, ayuda en la comprensión de explicación cuantitativa y otros.

Además, en el caso de la utilización de las preguntas intercaladas y las ilustraciones en esta clasificación tienen la mayor frecuencia, siendo un resultado similar a lo obtenido por Tapia y Arteaga (2008), cuando manifiestan que estás fueron las más aplicadas por los docentes en clases de biología.

Tabla 3. Parte II: Tipos de estrategias de enseñanza utilizadas por docentes de media general.

\begin{tabular}{|c|c|c|c|c|c|}
\hline \multirow[b]{2}{*}{ Estrategias } & \multicolumn{4}{|c|}{ Escala de valor } & \multirow{2}{*}{ Media } \\
\hline & Siempre & $\begin{array}{c}\text { Algunas } \\
\text { veces }\end{array}$ & $\begin{array}{c}\text { Pocas } \\
\text { veces }\end{array}$ & Nunca & \\
\hline \multirow{3}{*}{$\begin{array}{l}\text { Mapas y redes } \\
\text { conceptuales }\end{array}$} & Fi: 15 & Fi: 5 & Fi: 2 & Fi: 2 & \multirow{3}{*}{3,10} \\
\hline & $\%: 63$ & $\%: 21$ & $\%: 8$ & $\%: 8$ & \\
\hline & Fr: 0,63 & Fr: 0,21 & Fr: 0,08 & Fr: 0,08 & \\
\hline \multirow{3}{*}{$\begin{array}{c}\text { Organizadores } \\
\text { gráficos }\end{array}$} & Fi: 6 & Fi: 6 & Fi: 6 & Fi: 6 & \multirow{3}{*}{2,42} \\
\hline & $\%: 25$ & $\%: 25$ & $\%: 25$ & $\%: 25$ & \\
\hline & Fr: 0,25 & Fr: 0,25 & Fr: 0,25 & Fr: 0,25 & \\
\hline \multirow{3}{*}{ Resumen } & Fi: 1 & Fi: 12 & Fi: 10 & Fi: 1 & \multirow{3}{*}{2,44} \\
\hline & $\%: 4$ & $\%: 50$ & $\%: 42$ & $\%: 4$ & \\
\hline & Fr: 0,04 & Fr: 0,50 & Fr: 0,42 & Fr: 0,04 & \\
\hline \multirow{3}{*}{$\begin{array}{l}\text { Organizadores } \\
\text { textuales }\end{array}$} & Fi: 9 & Fi: 7 & Fi: 4 & Fi: 4 & \multirow{3}{*}{2,71} \\
\hline & $\%: 38$ & $\%: 29$ & $\%: 17$ & $\%: 17$ & \\
\hline & Fr: 0,38 & Fr: 0,29 & Fr: 0,17 & Fr: 0,17 & \\
\hline
\end{tabular}

Fuente: Elaboración propia

En la tabla número 3 es posible apreciar que los datos obtenidos para las estrategias con mapas y redes conceptuales tuvieron un porcentaje del $63 \%$ para la opción siempre con una media de 3,10 , que con respecto con el baremo representa una categoría alta en su utilización. Por su parte, los organizadores gráficos tienen un porcentaje igual para cada una de las opciones con un $25 \%$ con una media del 2,42 ubicada en la categoría alta. Mientras que los organizadores textuales, con un $38 \%$ para la opción siempre y $17 \%$ para pocas veces y nunca, con una media de 2,42 
ubicada en la categoría alta. Y, el resumen, tuvo una mayor frecuencia en la opción algunas veces con el $50 \%$ seguido de un $42 \%$ para pocas veces, tendiendo una media de 2,44 .

Cada una de estas categorías estrategias estás asociadas a estrategias para organizar la información nueva por aprender, como bien señalan Díaz y Hernández (2002), estás ayudan a estructurar adecuadamente la información. En este sentido, cada una sirve para organizar el contenido científico escolar de tal manera los estudiantes pueden comprender de mejor manera el tema. Además, permiten sintetizar el contenido en puntos relevantes o mostrar la información en partes claves para luego su compresión sea en un nivel mayor.

Bajo este contexto, la aplicación de estas estrategias de enseñanza en las clases de evolución, como el caso de utilizar los mapas y redes conceptuales contribuye al entendimiento de contenidos que pueden resultar en ocasiones abstractos para los estudiantes. A la par, dichas estrategias tienen para esta investigación el mayor valor de media aritmética, por ello se acota que estás tienen a facilitar el proceso de enseñanza puesto que es posible mostrar una abstracción, distribución de conceptos, ejemplos, postulados, enunciados, biografías, y demás durante la dinámica de una clase.

Tabla 5. Parte III: Tipos de estrategias de enseñanza utilizadas por docentes de media general

\begin{tabular}{|c|c|c|c|c|c|}
\hline \multirow[b]{2}{*}{ Estrategias } & \multicolumn{4}{|c|}{ Escala de valor } & \multirow{2}{*}{ Media } \\
\hline & Siempre & $\begin{array}{c}\text { Algunas } \\
\text { veces }\end{array}$ & $\begin{array}{c}\text { Pocas } \\
\text { veces }\end{array}$ & Nunca & \\
\hline \multirow{3}{*}{$\begin{array}{c}\text { Organizadores } \\
\text { previos }\end{array}$} & Fi: 13 & Fi: 5 & Fi: 5 & Fi: 1 & \multirow{3}{*}{3,00} \\
\hline & $\%: 54$ & $\%: 21$ & $\%: 21$ & $\%: 4$ & \\
\hline & Fr: 0,54 & Fr: 0,21 & Fr: 0,21 & Fr: 0,04 & \\
\hline \multirow{3}{*}{ Señalizaciones } & Fi: 6 & Fi: 9 & Fi: 9 & Fi: 0 & \multirow{3}{*}{2,70} \\
\hline & $\%: 25$ & $\%: 38$ & $\%: 38$ & $\%: 0$ & \\
\hline & Fr: 0,25 & Fr: 0,38 & Fr: 0,38 & Fr: 0 & \\
\hline \multirow{3}{*}{ Analogías } & Fi: 15 & Fi: 7 & Fi: 2 & Fi: 0 & \multirow{3}{*}{3,23} \\
\hline & $\%: 63$ & $\%: 29$ & $\%: 8$ & $\%: 0$ & \\
\hline & Fr: 0,63 & Fr: 0,29 & Fr: 0,08 & Fr: 0 & \\
\hline
\end{tabular}

Fuente: Elaboración propia 
Eloy León

Telos Vol. 21, No. 1 (2019). 144-165

Las señalizaciones presentaron un 38\% para las opciones de algunas veces y pocas veces, con una media del 2,7 estando en la categoría alta. Por su parte, los organizadores previos tienen un $54 \%$ para opción de siempre y un $21 \%$ para las opciones algunas veces y pocas veces, presentando una media de 3,00, este resultado las ubica en la categoría alta. Por último, las analogías tienen un porcentaje mayor para la opción siempre con un $63 \%$ y una media de 3,23, ello la ubica en la categoría muy alta.

Las señalizaciones están representadas como Estrategias para guiar la atención y el aprendizaje. Las analogías y los organizadores previos, están asociadas a Estrategias para promover el enlace entre los conocimientos previos y la nueva información que ha de aprender. Vale resaltar, que los organizadores textuales pueden incluirse en esta referencia.

La utilización de las señalizaciones, analogías y organizadores previos en clases de biología concuerda con lo reseñado por Tapia y Arteaga (2009). Lo cual permite al estudiante realizar una autoevaluación de forma gradual. Además, en el caso de ese estudio, así como los resultados de esta tabla, las analogías fueron muy aplicadas. Lograr la atención del estudiante, durante el proceso de enseñanza es de suma importancia, puesto que posibilita estructurar los conceptos nuevos a aprender.

\section{Consideraciones finales}

Cada una de las estrategias de enseñanza aplicadas no sólo deben aparecer en el papel de la planificación escolar, sino que tienen que ser utilizadas adecuadamente, porque estas son comúnmente actividades para promover, beneficiar, ampliar o profundizar, habilidades de destrezas cognitivas y motrices, ayudar a una observación aguda, ser críticos, objetivos, en el caso de las ciencias biológicas, comprender el estudio de los fenómenos naturales, entre otros.

En virtud de ello, el docente debe buscar estrategias muchas veces acorde al entorno cultural o social de los estudiantes, de tal manera estén acordes al momento de 
la enseñanza. Ciertamente, cada grupo de alumnos, así como cada persona, pueden llegar a presentar características distintas, por ello realizar en algunas ocasiones una exploración y escuchar o leer las ideas previas, tiende a ser eficaz.

Ahora bien, según la pregunta formulada en el presente trabajo de investigación sobre la frecuencia de las estrategias de enseñanza en clases de evolución biológica, se determinó lo siguiente:

La estrategia que tiene la mayor media aritmética fue las ilustraciones seguidamente por las preguntas intercaladas, analogías y mapas y redes conceptuales. En la revisión de los antecedentes, en el discurso oral de los profesores aplicar las preguntas intercaladas es muy común para las clases de biología. En este sentido, siendo el caso de la evolución biológica genera muchas interrogantes por parte de los estudiantes, es habitual que los docentes utilicen dichas estrategias.

Las ilustraciones y las preguntan intercaladas, tuvieron el mayor porcentaje para la opción Siempre. Además, son estrategias que pueden estar representadas como estrategias para mejorar la codificación de la información a aprender. A la par, vale acotar que dependiendo el criterio clasificar o catalogar las estrategias, estas pueden estar en una u otra clasificación.

Por otro lado, las gráficas presentaron el mayor porcentaje para la opción Nunca y con una media aritmética más baja. Evidentemente, para este estudio las gráficas no son muy aplicadas, a pesar de que existen contenidos de la biología evolutiva en las cuales pueden utilizarse ejercicios con gráficas.

Por último, para el caso de esta muestra de profesores de media general o bachillerato, los resultados indican que estos aplican una tendencia alta de estrategias de enseñanza para lograr las competencias académicas o al menos la comprensión de la evolución biológica.

\section{Referencias bibliográficas}

Acosta, Savier y Boscan, Adriana. (2012). Estrategias cognoscitivas para la promoción del aprendizaje significativo dela Biología, en la Escuela de Educación. Revista Telos. Vol. 14, No. 2. Venezuela. (Pp. 175-193). 
Ausubel, David; Novak, Josef; y Hanesian, Hortencia. (1991). Psicología educativa. Un punto de vista cognoscitivo. Editorial Trillas. México.

Anijovich, Rebeca y Mora, Silvia. (2009). Estrategias de enseñanza: Otra mirada al quehacer en el aula. Editorial AIQUE. Argentina.

Campos, Yolanda. (2000). Estrategias didácticas apoyadas en tecnología. DGENAMDF. México. Extraído de: http://www.camposc.net/0repositorio/libros/estrategias/libroEstrategias.html consultado: 05/06/2018.

Díaz, Frida y Hernández, Gerardo. (2002). Estrategias docentes para un aprendizaje significativo: una interpretación constructivista (segunda edición). Editorial Mc Graw Hill. México.

Futuyma, Douglas. (2005). Evolution. Sinauer Associates. USA.

García, Juan y Cañal de León, Pedro. (1995). ¿Cómo enseñar? Hacia una definición de las estrategias de enseñanza por investigación. Revista Investigación en la Escuela. No. 25. España. (Pp. 5-16).

González Galli, Leonardo. (2012). Enseñar y aprender biología evolutiva en el siglo XXI. Revista Bio-grafia: Escritos sobre la biología y su enseñanza. Monográfico de Evolución. Vol. 5, No. 9. Julio-diciembre. Colombia. (Pp. 1$3)$.

Hurtado de Barrera, Jacqueline. (2012). Metodología de la investigación: Guía para la compresión holística de la ciencia (cuarta edición). Editorial Quirón. Venezuela.

Hernández, Roberto., Fernández, Carlos y Baptista, María del pilar. (2014). Metodología de la investigación (sexta edición). Editorial Mc Graw Hill. México.

León, Eloy. (2017). Enseñanza de la evolución biológica. Una mirada al estado de la episteme docente. II Jornadas de investigación e innovación educativa. I Internacionales. Universidad nacional abierta. Centro local Zulia. Maracaibo. Venezuela.

León, Eloy y Morales, Marbett. (2017). Experiencia didáctica: Las tic en la enseñanza de la evolución biológica. Revista Eduweb. Vol. 11. No 1. (Pp. 101-112).

Martínez, Elizabeth y Zea, Enilda. (2004). Estrategias de enseñanza basadas en un enfoque constructivista. Revista Ciencias de la Educación. Vol. 2, No. 24. Julio-diciembre. Venezuela. (Pp. 69-90).

Mclnerney, Joseph. (2009). La enseñanza de la evolución siglo y medio después de El origen de las especies. Revista Ciencia Hoy. Vol. 19, No113. Argentina. (Pp. 76-83).

Pimienta, Julio. (2012). Estrategias de enseñanza-aprendizaje. Docencia universitaria basada en competencias (primera edición). Editorial Pearson. México.

Ruiz Gutiérrez, Rosaura., Álvarez, Eréndira., Noguera, Ricardo y Esparza, Martha. (2012). Enseñar y aprender biología evolutiva en el siglo XXI. Revista Bio- 
grafia: Escritos sobre la biología y su enseñanza. Monográfico de Evolución. Vol. 5, No. 9. Julio-diciembre. Colombia. (Pp. 80-88).

Tapia, Fernando y Arteaga, Yannett. (2009). Estrategias para la enseñanza de la célula aplicadas por docentes de educación básica. Revista Educare.Vol.13, No. 1. Venezuela. (Pp. 97-120).

Vera, Adriana y Vera, Luis. (2011). Estrategias utilizadas por los docentes para promover el aprendizaje de la biología a nivel universitario. Revista Telos. Vol. 13, No. 3. Septiembre-diciembre. Venezuela. (Pp. 397-411). 